\title{
Das Beste aus beiden Welten ${ }^{1}$
}

von Boris Jacob und Jin Tan

$\mathrm{D}$ er durch die Digitalisierung der Medien und die fortschreitende Vernetzung der Kommunikationswege eingeleitete Paradigmenwechsel im Umgang mit Medien ${ }^{2}$ stellt die Institution Bibliothek schon seit einiger Zeit vor neue Herausforderungen. Dabei ist die derzeitige Medienrevolution bei weitem nicht die erste, die eine Evolution der Bibliothek bewirkt. Einer der zentralen Aspekte dieses Prozesses, der Übergang vom Realen ins Virtuelle, führt einerseits zu Veränderungen unter der Fragestellung inwieweit die Bibliothek bei der Vermittlung ihrer Dienstleistungen noch an ein Gebäude gebunden ist. Andererseits wird die Diskussion nun um den Aspekt der veränderten Kommunikationsmöglichkeiten erweitert.

Während viele Medienproduzenten zu spät auf die veränderten Bedingungen der Internetökonomie reagiert haben, sind die Bibliotheken schon länger damit beschäftigt, ihre Angebote auf das Internet auszuweiten. Dazu wurden Kataloge digitalisiert, Zugang zu Datenbanken angeboten, digitale Bibliotheken aufgebaut und zunehmend beginnen auch deutsche Bibliotheken Bücher im großen Maßstab zu digitalisieren - mit dem Ziel, die Position der Bibliothek, die sie als Informationseinrichtung lange Zeit in der Mitte der Gesellschaft einnahm, zu verteidigen. Libraries are in the heart of the information society, so drückte es die ehemalige IFLA Präsidentin Kay Raseroka bei der IFLA- Konferenz in Berlin aus. Damit die Bibliothek dieser vielfältigen Anforderungen auch weiterhin gerecht wird, ist eine Anpassung an die sich ständig verändernde Umwelt notwendig. Festzuhalten ist, dass die Bibliothek nicht länger nur Das Buch und sein Haus ist ${ }^{3}$. T. Scott Plutchak ist sogar der Meinung, dass ihre Bedeutung als zentrale Medien- und Informationseinrichtung in der Zukunft weiter abnehmen wird:

"In the print world, the library building was the center of the librarian's universe; now, it may be a home base, but it is no longer where we necessarily do our most vital work." ${ }^{4}$

Wenn sich das Betätigungsfeld der Bibliotheken aber in den virtuellen Raum ausweitet, muss sie sich nicht nur der sich immer schneller wandelnden technischen Möglichkeiten bewusst sein, sondern auch ihrer Konkurrenz. Im Internet haben sich längst Gatekeeper etabliert, deren Kerngeschäft das Annotieren und Anzeigen digitaler Information ist, Google ist ein Beispiel dafür. Den Kampf gegen diese Konkurrenz auf ihrem eigenen Territorium aufzunehmen, so wie es Jean-Noël Jeanneney vorgeschlagen hatte ${ }^{5}$, wäre zwar eine Möglichkeit, die finanziellen Mittel, die für Personal, Reorganisation und Technik, aufgebracht werden müssten aber wären enorm. Eine andere Möglichkeit ist es, von der Konkurrenz zu lernen und da, wo es möglich ist, mit ihr zu kooperieren. Dabei hat gerade die Bibliothek die Chance, Angebote zu entwickeln und

\footnotetext{
${ }^{1}$ Die wörtliche Übersetzung des Titels der Star Trek Serie TNG 74 u. 75: „The Best of Both Worlds“. Die deutschen Titel „In den Händen der Borg“ und „Angriffsziel Erde“ hätten den Inhalt unseres Artikels nicht ganz getroffen.

${ }^{2}$ Eine Studie zeigt bspw. dass die Nutzung des Internets von amerikanischen Erwachsenen in den letzten zehn Jahren von $16 \%$ auf $70 \%$ angestiegen ist:

http://online.wsj.com/public/article/SB114840389678260791IREjYVgN_rGLeE3_6Djin1jeJZc_20070523.html

${ }^{3}$ Schöne Beispiele für „Das Buch und sein Haus“ finden sich auf: www.bibliotheksbauten.de/

${ }^{4}$ http://tscott.typepad.com/tsp/2007/01/what_do_you_cal.html

${ }^{5}$ Siehe dazu das Plädoyer des ehemaligen Präsidenten der Bibliothèque National de France: Jeanneney, Jean-Noël

(2006): Googles Herausforderung : Für eine europäische Bibliothek.
} 
anzubieten, die langfristig, sicher und öffentlich zugänglich und jenseits des Perpetual Beta sind, und gleichzeitig neue Wege zu finden, um ihre traditionellen Dienstleistungen den technischen Gegebenheiten anzupassen und so attraktiver zu machen.

Um dieser Aufgabe nachzukommen, reicht es aber für Bibliotheken inzwischen nicht mehr aus, nur auf die „Portalsmentalität“ zurückzugreifen, die sich sowohl auf das Gebäude Bibliothek als auch auf deren Internetangebot bezieht. Denn wenn der Nutzer nicht zum Portal kommt...:

"One of the things I am concerned about is when I hear people talking about 'What can we do in order to get people into the library?' As if the goal is to get people into the library. And I think that's the wrong goal. The goal is to connect people with knowledge and information, ideas and the work that other people have done."

Entscheidend ist aus diesem Blickwinkel nicht mehr das physische Gebäude, in dem Bibliothekare arbeiten, entscheidend ist letztendlich die kreative Arbeit der Bibliothekare selbst. Um möglichst kundenorientiert zu sein, wird sich die Bibliothek dabei von einer Just-In-Case- zu einer Just-In-Time- Bibliothek entwickeln müssen ${ }^{7}$ und dabei sowohl ihre physische als auch ihre digitale Form neu überdenken. Indem sich das $W W W$ unter der Bezeichnung Web 2.0 langsam zu dem entwickelt, als das es ursprünglich von Tim Berners-Lee konzipiert wurde, nämlich zu einem Kommunikations- und Kollaborationsmedium, ${ }^{8}$ können die Konsumenten von Information gleichzeitig als Produzenten in Erscheinung treten. So kürte das Time Magazine im letzten Jahr den Internetnutzer auch als Person des Jahres: Yes, you. You control the Information Age. Welcome to your world. ${ }^{9}$

Der Begriff Bibliothek 2.0 ist in diesem Zusammenhang Mittel zum Zweck: die Bibliothek, die bibliothekarische Kompetenz und die bibliothekarischen Konzepte unter diesen veränderten Bedingungen zu diskutieren. Sicherlich kann man wie Signy Irene Karlsen fragen, ob es sich dabei um "Same shit, new wrapping?"10 handelt, eine differenzierte Betrachtung ist dennoch notwendig. Ob das allerdings ausreicht, den Platz in der Mitte der Wissensgesellschaft zu verteidigen, ist fraglich. Die große Chance der Bibliothek besteht darin, zukunftsweisende Services zu entwickeln. Dies muss auf der Basis des bibliothekarischen Wissens im Umgang mit Information und im Zusammenwirken mit den immer neuen Technologien und den dadurch entstehenden Möglichkeiten geschehen. Technisch ist viel mehr möglich, als bisher umgesetzt wurde. Dorthin zu gehen, wo die Benutzer sind, ist einer von vielen Vorschlägen von Laura B. Cohen: ${ }^{11}$ Bibliothekare müssen einerseits ihre traditionelle Arbeit als Informationsspezialisten oder -vermittler im Hinblick der technischen Entwicklung wie z. B. des Web 2.0 verbessern und andererseits ihr Umfeld beobachten, um den Bibliotheksservice an die Bedürfnisse ihrer Kunden

\footnotetext{
${ }^{6}$ The Library 2.0 Gang talk about changing library buildings: http://talk.talis.com/archives/2007/01/the_library_20_7.html

${ }^{7}$ Danowski, Patrick; Heller, Lambert (2006) Bibliothek 2.0 - Die Zukunft der Bibliothek? Zitiert nach: Hobohm, Hans-Christoph (1997) Auf dem Weg zur lernenden Organisation. In: Bibliothek, Heft 21, S. 293. - URL: http://eprints.rclis.org/archive/00007618/

${ }^{8}$ www-128.ibm.com/developerworks/podcast/dwi/cm-int082206txt.html

${ }^{9}$ www.time.com/time/covers/0,16641,20061225,00.html

${ }^{10}$ Karlsen, Signy Irene (2006) The Public Library. A New Version. In: Scandinavian Public Library Quarterly 3, S. 4-6

${ }^{11}$ http://bibliothek2.wordpress.com/2006/11/13/manifest-des-bibliothekars-20-dt-ubersetzung/
} 
anzupassen. Michael Stephens beschreibt dies in seinen zehn Trends zur Bibliotheksarbeit im Jahr 2007: Conversations, Convergence, Content, Redefining LIS Jobs, Citizen Journalism, We're Human, Openess \& Sharing, Participation, Experience, Play. ${ }^{12}$

Trotzdem ist zu hinterfragen, bis zu welchem Grad T. Scott Plutchaks These über den Bedeutungsverlust der physischen Bibliothek zutreffend ist, denn die Dependancen einiger Bibliotheken in der kommerziellen virtuellen Welt Second Life ${ }^{13}$ oder Buchbetrachtungsprogramme wie Turning the Page $2.0^{14}$, als Beispiele für die virtuelle Ordnung und Darstellung von Wissen, werden die Bibliothek als realen, öffentlichen und kulturellen Ort nicht so einfach ersetzen können. Jürgen Seefeldt drückt diese Idee passend aus:

“Als Kontrapunkt zur virtuellen Welt, dem Cyberspace, könnte die neue, reale Bibliothek von morgen an jedem Ort der Welt den Bezugspunkt mit Wissenszugang, persönlicher Betreuung und Chancengleichheit aller darstellen, sozusagen einen gemeinsamen Identifikationsrahmen bieten und so dem seit alters her bestehenden und weiterhin wachsenden Bedürfnis nach Gemeinschaft entsprechen [...]."15

Die Verwendung des Wortes „könnte“ impliziert, dass sich Bibliotheken im Real Life nicht so wahrnehmen. Ein Umdenken und eine kreative Auseinandersetzung findet aber auch unter diesem Aspekt statt. Beispiel dafür, dass sich die Bibliothek neu in die Stadtgesellschaft einordnet sind die Ideastores in England ${ }^{16}$, die einerseits bibliothekarische Dienste unter einem anderen Image anbieten, andererseits neben Büchern aber auch Cafés, Trainingsräume und andere Räume und Möglichkeiten für das öffentliche Leben bereithalten. Ein abstrakteres und schwer zu realisierendes Beispiel ist die Brabant-Bibliothek, ein Turm, bestehend aus einer spiralisch in die Höhe ragenden Aneinanderreihung von Bücherregalen. Dieses Gebäude, vielleicht ist es passender von einem Weg zu sprechen, hat die Funktion einer Zentralbibliothek, die für die Literaturversorgung einer Vielzahl von verschiedenen Einrichtungen aller Art verantwortlich ist. ${ }^{17}$ Denkbar sind Schulen, Cafés oder Kultureinrichtungen.

Letztendlich wird es darum gehen, die Vorteile aus Virtualität und Realität in der Bibliothek zu vereinen. Und so spielt beim Projekt Second Library 2.0 auch die Realität eine Rolle:

"The goal of these projects is to put the library where the user is - on the Internet - to provide vital services there and also to promote the local library."18

Dafür gibt es bereits viele gute Ideen und erfolgreich funktionierende Konzepte. Dank dem technologischen Fortschritt war es noch nie so einfach, diese fruchtbar zu vernetzen.

\footnotetext{
12 http://tametheweb.com/2007/02/ten_tech_trends_for_librarians_1.html

${ }^{13}$ Jin Tan listet solche in seinem Blog: http://jintan.wordpress.com/2007/03/21/bibliotheken-in-second-life/

${ }^{14}$ Das Programm entstand in Zusammenarbeit der British Library mit Microsoft: www.bl.uk/ttp2/ttp2.html. 15 Seefeldt, Jürgen: Zukunftsvisionen : Die Bibliothek von morgen - URL: www.b-i-t-online.de/archiv/2005 01/fach1.htm

${ }^{16}$ Ein Konzeptpapier dazu gibt es auf: www.ideastore.co.uk/downloads/A_Library_and_Lifelong_Learning_ Development_Strategy_for_Tower_Hamlets.pdf

${ }^{17}$ Bibliotheken 2040: Die Zukunft neu entwerfen / Red. Rob Bruinzeels und Nicole van Tiggelen. Übers. von Uta Klaassen. - Bad Honnef: Bock + Herchen, 2003, S. 16ff.

${ }^{18}$ www.talis.com/tdn/node/1506
} 